\title{
HIGH-CONTRAST NEAR-INFRARED POLARIZATION IMAGING OF MWC480
}

\author{
N. Kusakabe ${ }^{1}$, C. A. Grady ${ }^{2}$, M. L. Sitko ${ }^{3,4,30}$, J. Hashimoto ${ }^{1}$, T. Kudo ${ }^{1}$, M. Fukagawa ${ }^{5}$, T. Muto ${ }^{6,7}$, J. P. Wisniewski ${ }^{8}$,

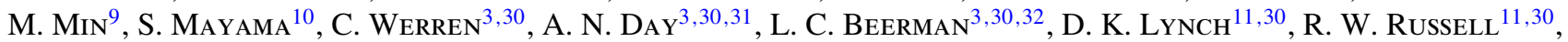 \\ S. M. BrafFord ${ }^{12,30}$, M. Kuzuhara ${ }^{13}$, T. D. Brandt ${ }^{14}$ L. AbE $^{15}$, W. Brandner ${ }^{16}$, J. CARSON $^{17}$, S. EGNER $^{18}$, M. FELDT $^{16}$,

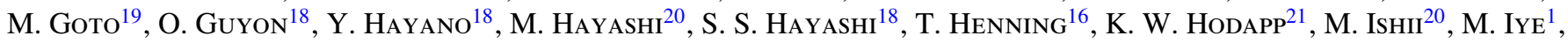 \\ M. JANSON ${ }^{14}$, R. KANDORI ${ }^{1}$, G. R. KNAPP ${ }^{14}$, T. MATSUO ${ }^{22}$, M. W. McElWAin ${ }^{23}$, S. MiYAma ${ }^{1}$, J.-I. Morino ${ }^{1}$, \\ A. Moro-Martin ${ }^{14,24}$, T. Nishimura ${ }^{18}$, T.-S. Pyo ${ }^{18}$, H. Suto ${ }^{1}$, R. Suzuki ${ }^{1}$, M. Takami ${ }^{25}$, N. TAKATO $^{18}$, H. Terada $^{18}$, \\ C. Thalmann ${ }^{26}$, D. Tomono ${ }^{18}$, E. L. Turner ${ }^{14,27}$, M. WATAnabe $^{28}$, T. YAmada $^{29}$, H. TAKami $^{18}$, T. Usuda $^{18}$, and M. TAmura $^{1}$ \\ ${ }^{1}$ National Astronomical Observatory, 2-21-1 Osawa, Mitaka, Tokyo 181-8588, Japan; nb.kusakabe@nao.ac.jp \\ ${ }^{2}$ Eureka Scientific, 2452 Delmer St., Suite 100, Oakland, CA 96402, USA \\ ${ }^{3}$ Department of Physics, University of Cincinnati, Cincinnati, OH 45221, USA \\ ${ }^{4}$ Space Science Institute, 4750 Walnut St., Suite 205, Boulder, CO 80301, USA \\ ${ }^{5}$ Department of Earth and Space Science, Graduate School of Science, Osaka University, 1-1, Machikaneyama, Toyonaka, Osaka 560-0043, Japan \\ ${ }^{6}$ Tokyo Institute of Technology, 2-12-1 Ookayama, Meguro, Tokyo 152-8551, Japan \\ ${ }^{7}$ Division of Liberal Arts, Kogakuin University, 1-24-2, Nishi-Shinjuku, Shinjuku-ku, Tokyo 163-8677, Japan \\ ${ }^{8}$ Department of Astronomy, University of Washington, Box 351580 Seattle, Washington, DC, USA \\ ${ }^{9}$ Astronomical Institute "Anton Pannekoek" Science Park 9041098 XH Amsterdam, The Netherlands \\ ${ }^{10}$ The Center for the Promotion of Integrated Sciences, The Graduate University for Advanced Studies, \\ Shonan International Village Hayama-cho, Miura-gun, Kanagawa, Japan \\ ${ }^{11}$ The Aerospace Corporation, Los Angeles, CA 90009, USA \\ 12 Brafford \& Phillips, Batavia, OH 45103, USA \\ ${ }^{13}$ Department of Earth and Planetary Science, The University of Tokyo, Hongo 7-3-1, Bunkyo-ku, Tokyo 113-0033, Japan \\ ${ }^{14}$ Department of Astrophysical Sciences, Princeton University, NJ 08544, USA \\ ${ }^{15}$ Laboratoire Lagrange, UMR7293, Université Le de Nice-Sophia Antipolis, CNRS, Observatoire de la Côte d'fAzur, 06300 Nice, France \\ ${ }^{16}$ Max Planck Institute for Astronomy, 69117 Heidelberg, Königstuhl 17, Germany \\ ${ }^{17}$ Department of Physics and Astronomy, College of Charleston, 58 Coming St., Charleston, SC 29424, USA \\ ${ }^{18}$ Subaru Telescope, 650 North A'hoku Place, Hilo, HI 96720, USA \\ ${ }^{19}$ Universitäts-Sternwarte München, Ludwig-Maximilians-Universität, Scheinerstr. 1, 81679 München, Germany \\ ${ }^{20}$ Department of Astronomy, The University of Tokyo, Hongo 7-3-1, Bunkyo-ku, Tokyo 113-0033, Japan \\ ${ }^{21}$ Institute for Astronomy, University of Hawaii, 640 North A'hoku Place, Hilo, HI 96720, USA \\ ${ }^{22}$ Department of Astronomy, Kyoto University, Kitashirakawa-Oiwake-cho, Sakyo-ku, Kyoto 606-8502, Japan \\ ${ }^{23}$ ExoPlanets and Stellar Astrophysics Laboratory, Code 667, Goddard Space Flight Center, Greenbelt, MD 20771, USA \\ ${ }^{24}$ Departamento de Astrofisica, CAB (INTA-CSIC), Instituto Nacional de Técnica Aeroespacial, Torrejón de Ardoz, 28850 Madrid, Spain \\ ${ }^{25}$ Institute of Astronomy and Astrophysics, Academia Sinica, P.O. Box 23-141, Taipei 106, Taiwan \\ ${ }^{26}$ Astronomical Institute “Anton Pannekoek," University of Amsterdam, Science Park 904, 1098 XH Amsterdam, The Netherlands \\ ${ }^{27}$ Institute for the Physics and Mathematics of the Universe, The University of Tokyo, Kashiwa 227-8568, Japan \\ ${ }^{28}$ Department of Cosmosciences, Hokkaido University, Sapporo 060-0810, Japan \\ 29 Astronomical Institute, Tohoku University, Aoba, Sendai 980-8578, Japan \\ Received 2012 April 23; accepted 2012 May 6; published 2012 June 25
}

\begin{abstract}
One of the key predictions of modeling from the IR excess of Herbig Ae stars is that for protoplanetary disks, where significant grain growth and settling has occurred, the dust disk has flattened to the point that it can be partially or largely shadowed by the innermost material at or near the dust sublimation radius. When the self-shadowing has already started, the outer disk is expected to be detected in scattered light only in the exceptional cases when the scale height of the dust disk at the sublimation radius is smaller than usual. High-contrast imaging combined with the IR spectral energy distribution allow us to measure the degree of flattening of the disk, as well as to determine the properties of the outer disk. We present polarimetric differential imaging in the $H$ band obtained with Subaru/HiCIAO of one such system, MWC 480. The HiCIAO data were obtained at a historic minimum of the NIR excess. The disk is detected in scattered light from 0.'2 to 1."0 (27.4-137 AU). Together with the marginal detection of the disk from 1998 February 24 by Hubble Space Telescope/NICMOS, our data constrain the opening half-angle for the disk to lie between $1.3 \leqslant \theta \leqslant 2.2$. When compared with similar measures in $\mathrm{CO}$ for the gas disk from the literature, the dust disk subtends only $\sim 30 \%$ of the gas disk scale height $(\mathrm{H} / \mathrm{R} \sim 0.03)$. Such a dust disk is a factor of 5-7 flatter than transitional disks, which have structural signatures that giant planets have formed.
\end{abstract}

Key words: protoplanetary disks - stars: individual (MWC 480) - stars: pre-main sequence

Online-only material: color figure

\section{INTRODUCTION}

For the vast majority of protoplanetary disks, modeling of the IR spectral energy distribution (SED) has been the principal

\footnotetext{
${ }^{30}$ Guest Observer, Infrared Telescope Facility.

${ }^{31}$ Now at Miami University, Oxford, OH 45056, USA.

32 Now at University of Washington, Seattle, WA 98195-1580, USA.
}

tool for characterizing the properties of the dust disk. Models for disks derived from such data are non-unique since the viewing geometry (inclination), the assumed grain opacity model, and the presence or absence of structure in the disk can combine to produce similar SEDs. Moreover, disks are not static: ISO and Spitzer studies of T Tauri stars have shown that variability in the 3-4.5 $\mu \mathrm{m}$ range is ubiquitous (Rebull et al. 2011; Kóspál et al. 
2012). At least some higher mass analogs to T Tauri stars, the Herbig Ae stars, also exhibit striking variability in the IR SED, which has been interpreted as reflecting changes in the scale height of the dust disk at or near the sublimation radius (Sitko et al. 2008).

For protoplanetary disks where there has been significant dust grain growth and settling, the dust disk may have flattened sufficiently to be partially or largely shadowed (Meeus et al. 2001; Dullemond \& Dominik 2004a, 2004b) by material at or near the dust sublimation radius. Under these circumstances, the amount of light reaching the outer disk is expected to be anticorrelated with the IR excess in the $1-10 \mu \mathrm{m}$ range, originating from material at or near the dust sublimation radius (Acke et al. 2009; Carciofi et al. 2004; Robitaille et al. 2007). Hubble Space Telescope (HST) and ground-based NIR single-epoch studies of Herbig Ae disks associated with IR SEDs interpreted as arising from shadowed disks (Meeus group II) resulted in a high non-detection rate, even for mm-bright, large protoplanetary disks (Grady et al. 2005; Fukagawa et al. 2010), but also in some surprising detections (Grady et al. 2000). Multi-epoch imaging of one of these stars, HD 163296, demonstrated that the illuminated portion of the disk varied with time (Wisniewski et al. 2008). These observations typically lacked simultaneous measurements of the IR SED, which would be needed to test whether the disk illumination is indeed anti-correlated with the near- and mid-IR light.

Another Herbig Ae star with documented near- and mid-IR excess variability is MWC 480 (HD 31648), located at $d=$ $137_{-21}^{+31} \mathrm{pc}$ (van Leeuwen 2007). This star is one of the brightest Herbig Ae stars at millimeter wavelengths (Mannings et al. 1997) and has been the subject of detailed studies from the X-ray through the radio regime (Grady et al. 2010; Simon et al. 2000; Piétu et al. 2006, 2007), as well as repeated high-contrast imaging with HST (Augereau et al. 2001; Grady et al. 2001, 2005, 2010) and NIR imaging with Subaru (Fukagawa et al. 2010). HST imaging at the epoch of ISO SWS observations resulted in a marginal scattered-light detection of this disk (Grady et al. 2010) at a time when the IR SED suggested that the IR excess was weaker than average. However, the disk detection rate in scattered light is known to increase as further steps are taken to increase the disk-to-star contrast, such as the use of polarimetric differential imaging, and as the inner working angle of the observations decreases. We present the first firm scattered light detection of the disk of MWC 480 with an inner working angle of 0.2 (27 AU).

\section{OBSERVATIONS AND DATA REDUCTION}

\subsection{Subaru/HiCIAO}

We carried out $H$-band $(1.6 \mu \mathrm{m})$ linear polarimetric observations of MWC 480 using the high-contrast imaging instrument HiCIAO (Tamura et al. 2006) with a dual-beam polarimeter at the Subaru $8.2 \mathrm{~m}$ Telescope on 2010 January 24. These observations were part of the Strategic Explorations of Exoplanets and Disks with Subaru (SEEDS; Tamura et al. 2009). The polarimetric observation mode acquires $o$-ray and $e$-ray simultaneously, and images a field of view of $10^{\prime \prime} \times 20^{\prime \prime}$ with a pixel scale of 9.53 mas pixel $^{-1}$. MWC 480 was observed using a circular occulting mask with $0^{\prime \prime} 3$ diameter. The exposures were performed at four position angles (P.A.s) of the half-wave plate, with a sequence of P.A. $=0^{\circ}, 45^{\circ}, 22.5$, and 67.5 to measure the Stokes parameters. The integration time per wave plate position was $19.5 \mathrm{~s}$ and we obtained 24 sets by repeating the cycle of the

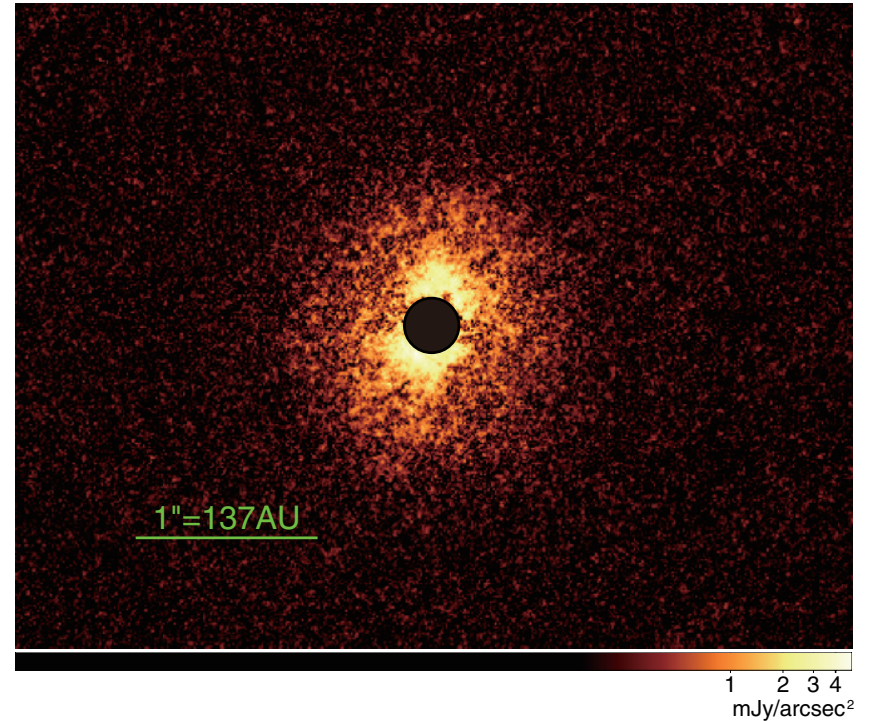

Figure 1. $H$-band PI image of MWC 480. The central black circle shown is the occulting mask ( $r=0^{\prime \prime}$. 15). North is up and east to the left in this image. (A color version of this figure is available in the online journal.)

wave plate. The adaptive optics system (AO188; Hayano et al. 2008) provided a diffraction limited and mostly stable stellar point-spread function (PSF) with an FWHM of 0 '.07 in the $H$ band. Low quality images were removed prior to production of the final images, resulting in 18 good data sets with a total integration time for the polarization intensity (hereafter PI) image of $351.0 \mathrm{~s}$. Note that the data sets are taken with Angular Differential Imaging mode, and the field rotation is $32^{\circ}$.

The polarimetric data reduction is described in Hashimoto et al. (2011) and Muto et al. (2012), using the standard approach for polarimetric differential imaging (Hinkley et al. 2009). Instrumental polarization of HiCIAO at the Nasmyth instrument was corrected by following Joos et al. (2008). We calculated PI from $\sqrt{Q^{2}+U^{2}}$. This approach mitigates two of the limitations of high-contrast imagery: the PSF observations are obtained simultaneously with the disk data, eliminating the effects of variable seeing, and also providing a PSF measurement which is a perfect color match to the star. This is particularly important for systems where the $J-H$ or $H-K$ colors are redder than any main-sequence candidates. While a PSF reference target was observed immediately after MWC 480, the seeing was gradually changing and the reference target data did not provide a good match to the PSF of the MWC 480 data. As a consequence, we focus on the PI image rather than the PSF subtraction intensity (I) image. The PI image is shown in Figure 1.

Non-saturated images of MWC 480 in the $H$ band were obtained immediately after the coronagraphic sequence, using an ND filter that transmits $10 \%$ of the light. Similar observations of the photometric calibration star HD 18881 (A0V, $J-H=$ $0.0^{m}=0.055, H-K=0^{m}$ ) were obtained on 2010 January 23. HD 18881 was selected from the UKIRT bright standard catalog (Hawarden et al. 2001) for the Mauna Kea filter set described by Simons \& Tokunaga (2002), Tokunaga et al. (2002), and Tokunaga \& Vacca (2005). We derived the zero-point magnitude as $24.8 \pm 0.01$ from the photometry of the calibration star.

\subsection{BASS Spectrophotometry}

We observed MWC 480 during six epochs between 1996 and 2010, using The Aerospace Corporation's Broadband Array Spectrograph System (BASS) on NASA's Infrared Telescope 
Table 1

SpeX Observations

\begin{tabular}{lcccc}
\hline \hline UT Date & Mode & MWC 480 Airmass & Calibration Star Airmass & Calibration Star \\
\hline 2006 Aug 22 & SXD & 1.14 & 1.15 & HD 25152 \\
& LXD & 1.19 & 1.15 & HD 25152 \\
2006 Aug 23 & SXD & 1.09 & 1.15 & HD 25152 \\
& LXD & 1.13 & 1.12 & HD 25152 \\
2006 Oct 19 & SXD & 1.04 & 1.06 & HD 31592 \\
& LXD & 1.04 & 1.07 & HD 31592 \\
2006 Nov 28 & SXD & 1.02 & 1.01 & HD 31592 \\
& LXD & 1.02 & 1.02 & HD 31592 \\
2007 Feb 25 & SXD & 1.18 & 1.06 & HD 31295 \\
& LXD & 1.24 & 1.37 & HD 31295 \\
2007 Dec 9 & SXD & 1.03 & 1.06 & HD 25152 \\
2007 Dec 10 & LXD & 1.28 & 1.11 & HD 25152 \\
& Prism & 1.05 & 1.06 & HD 31069 \\
2008 Oct 4 & SXD & 1.12 & 1.21 & HD 31069 \\
& LXD & 1.09 & 1.23 & HD 31069 \\
& Prism & 1.04 & 1.01 & HD 31069 \\
2009 Dec 1 & SXD & 1.20 & 1.16 & HD 31069 \\
& LXD & 1.16 & 1.19 & HD 31069 \\
& Prism & 1.70 & 1.66 & HD 31069 \\
& SXD & 1.04 & 1.11 & HD 25152 \\
& SXD & 1.02 & 1.07 & 1.14 \\
& LXD & 1.12 & 1.05 & \\
\hline
\end{tabular}

Facility (IRTF). BASS uses a cold beamsplitter to separate the light into two separate wavelength regimes. The shortwavelength beam includes light from 2.9 to $6 \mu \mathrm{m}$, while the long-wavelength beam covers 6-13.5 $\mu \mathrm{m}$. Each beam is dispersed onto a 58-element blocked impurity band linear array, thus allowing for simultaneous coverage of the spectrum from 2.9 to $13.5 \mu \mathrm{m}$. The spectral resolution $R=\lambda / \Delta \lambda$ is wavelength dependent, ranging from about 30 to 125 over each of the two wavelength regions (Hackwell et al. 1990). The entrance aperture of BASS is a $1 \mathrm{~mm}$ circular hole, whose effective projected diameter on the sky was 3".3-3".4, while a redesign of the dewar optics increased this value to 4". 0 in 2010. The observations are calibrated against spectral standard stars located close to the same airmass. Due to its proximity in the sky, $\alpha$ Tau usually serves as the flux calibration star.

\subsection{SpeX Cross-dispersed Observations}

We also observed MWC 480 with the SpeX spectrograph on IRTF on 11 nights between 2006 and 2011 (Table 1), extending the study of Sitko et al. (2008). The SpeX observations were made using the cross-dispersed (XD) echelle gratings in both short-wavelength mode (SXD), covering 0.8-2.4 $\mu \mathrm{m}$, and longwavelength mode (LXD), covering 2.3-5.4 $\mu \mathrm{m}$ (Rayner et al. 2003,2009 ). These observations were obtained using a $0{ }^{\prime} 8$ wide slit, corrected for telluric extinction, and flux calibrated against a variety of A0V calibration stars observed at airmasses close to that of MWC 480. The data were reduced using the Spextool software (Vacca et al. 2003; Cushing et al. 2004) running under IDL.

Due to the light loss introduced by the $0{ }^{\prime} 8$ slit used to obtain the SXD and LXD spectra, changes in telescope tracking and seeing between the observations of MWC 480 and a calibration star may result in merged SXD or LXD spectra with a net zeropoint shift compared to their true absolute flux values. We used a variety of techniques to check for any systematic zero-point shift in the absolute flux scale, as discussed below in greater detail. These included using the low dispersion prism in SpeX with a 3.0 wide slit, $J H K$ photometry with the SpeX guider, and the $3-13.5 \mu \mathrm{m}$ spectrophotometry from BASS.

\subsection{SpeX Prism Observations}

On many nights, we observed MWC 480 and its A0V calibration star using the low-dispersion Prism and a 3".0 wide slit. To avoid saturation of the detector and minimize the wavelength calibration arc line blends, the flat-field and wavelength calibration exposures required a narrower slit, and the 0!'8 slit was used. On nights of good transparency and seeing of $1^{\prime \prime} .0$ or better, this generally produces the same flux calibration as the other methods. On such nights, very little light hit the slit jaws, and individual exposures showed minimal scatter. However, during nights of exceptionally good seeing ( 0 '.7 or better), motion of the star on the detector can introduce small wavelength shifts, which will result in undercorrected or overcorrected telluric bands with a characteristic "P Cygni" shape. These have a minimal affect on the overall absolute flux calibration, however.

\subsection{Near-IR Photometry}

On the same night that some of the spectra of MWC 480 were obtained with SpeX, images in the $J, H$, and $K$ filters of the SpeX guider camera ("Guidedog") were obtained. This uses the Mauna Kea filter set.

Three calibration stars were also observed. Two of these, HD289907 and SA-97-249, are standard stars for the Mauna Kea filter set, and the magnitudes of Leggett et al. (2006) were used. The third was HD 31069, the A0V star used for the some of the spectroscopy, where Two Micron All Sky Survey (Skrutskie et al. 2006; Cutri et al. 2003) values were used. Even with short $(0.12 \mathrm{~s})$ integration times per image, slight saturation effects were evident in some of the $K$-band data, and these were not used for the absolute flux calibration. 


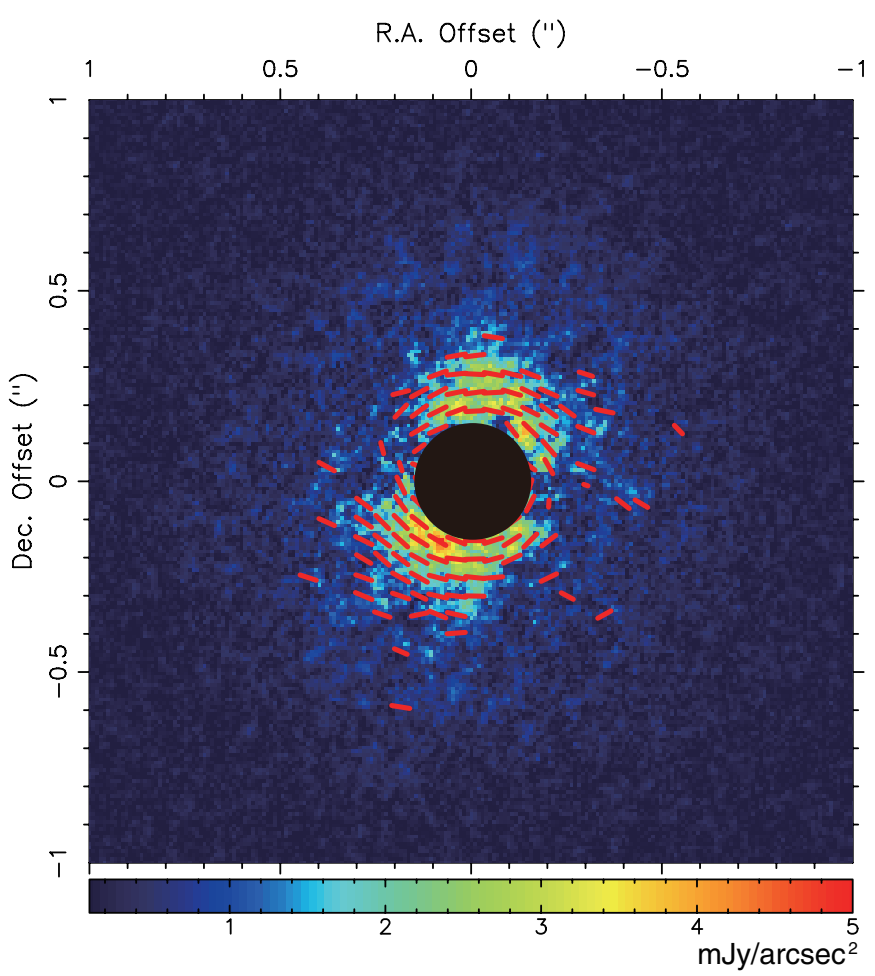

Figure 2. Polarization vector map superposed on the $H$-band PI image of MWC 480. The central black circle shown is the occulting mask $\left(r=00^{\prime \prime} 15\right)$. The vectors show just polarization angle, not polarization degrees.

\section{RESULTS}

\subsection{Disk Polarization}

The disk of MWC 480 is detected out to a radius of $\sim 1^{\prime \prime}$ (137 AU) (Figure 1). The central black circle indicates the 0 .' 15 radius of the occulting mask. We measure an integrated PI from 0.2 to $2 . .0$ of $1.2 \pm 0.1 \mathrm{mJy}$, with $0.6 \pm 0.1 \mathrm{mJy}$ exterior to 0 .' 6 in radius. On the same night, the star was at $H=6.74 \pm 0.08$ mag, resulting in a polarized fractional intensity of $0.08 \%$. In contrast to the NICMOS marginal detection (Grady et al. 2010), the disk is detected at all position angles, except for a dark lane along P.A. $\sim 60 \pm 10^{\circ}$. This P.A. is in reasonable agreement with the average disk semiminor axis position angle of $57^{\circ} \pm$ 2.2 derived from millimeter interferometry for an average of five $\mathrm{CO}$ and $\mathrm{HCO}^{+}$transitions (Piétu et al. 2007) and the P.A. of the jet (Grady et al. 2010). We therefore interpret the dark band as a feature caused by depolarization due to the grain scattering phase function, with no indication of material deficits in the disk (Min et al. 2012; Tanii et al. 2012).

The vector map of the PI disk is shown in Figure 2, superposed on the bright PI disk. The vectors show only polarization angle information, since we have no contemporary $I$ data. The bright inner disk $(0.2 \leqslant r \leqslant 0.4)$ polarization vectors show centro-symmetric features (e.g., a butterfly pattern), indicating that the PI disk is really scattered light from the central star.

\subsection{Polarization Intensity Radial Profile}

In common with other SEEDS studies of young stellar object disks, we measure the disk radial polarized surface brightness (SB) profile along the disk major axis, or the axis of maximum elongation in the HiCIAO data. We extracted a swath 0.'13 wide $(2 \times$ the PSF FWHM) along the major axis from the center of

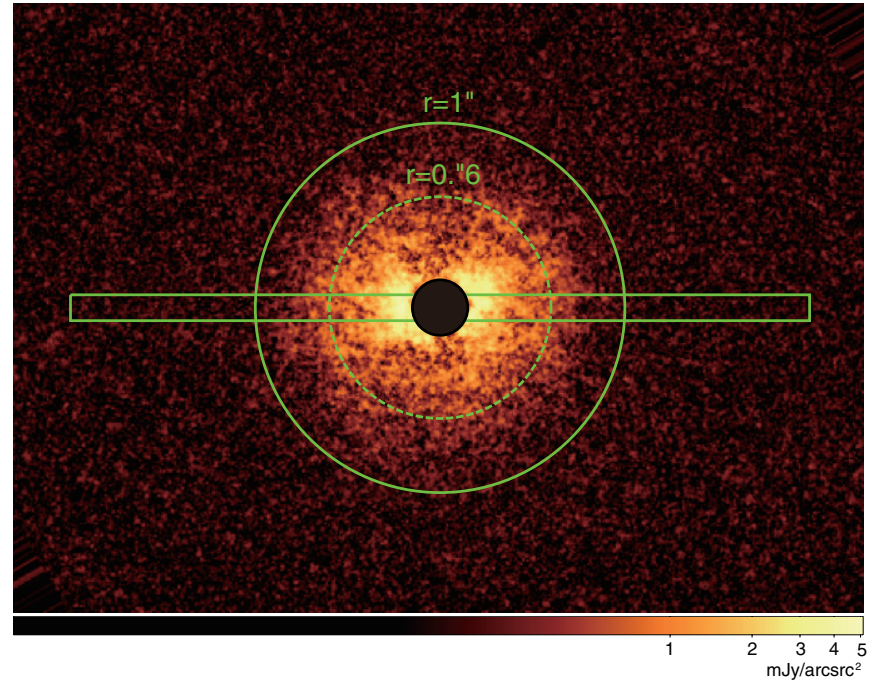

Figure 3. Rotated image of polarization intensity radial profile of MWC 480. The vertical and horizontal P.A.s are $60^{\circ}$ and $150^{\circ}$. The green box is the position of radial profile, swath 14 pixel width. The black circle shows the occulting mask $\left(r=0^{\prime \prime} 15\right)$.

the coronagraphic spot out to $3^{\prime \prime}$ on both sides of the star. PI brightness was measured in 14 pixel $\times 1$ pixel, $0^{\prime \prime} 13 \times 0^{\prime \prime} .01$ (Figure 3). Both radial profiles are shown in Figure 4 . In the profile, $r=0$ '. 15 is the occulting mask edge.

From 0.2 to 0.6 (27-84 AU) we find that the PI radial $\mathrm{SB}$ of P.A. $=150^{\circ}$ and $30^{\circ}$ drops as $r^{-1.7 \pm 0.1}$ and $r^{-2.0 \pm 0.1}$, respectively. From 0'.6 to $1^{\prime \prime} 0$ (84-137 AU), the radial SB profile is steeper, $r^{-3.0 \pm 0.3}$. The outer disk radial SB profile is similar to that of HD 169142 from 123 to 200 AU (Fukagawa et al. 2010; Grady et al. 2007), HD 100546 from 10 to 140 AU (Quanz et al. 2011), and SAO 206462 (Muto et al. 2012). The shallow radial SB profile from 0.'2 to 0.'6 is similar to that seen in SAO 206462 in the region of the spiral arms where the polarization fractional intensity is $0.6 \%$ (Muto et al. 2012), although no resolved spiral features are detected in the disk of MWC 480 at the resolution of our data.

\subsection{Placing the HiCIAO Detection in Context}

MWC 480 has a history of correlated near- and mid-IR variability (Sitko et al. 2008) with changes in the amplitude of the IR excess, rather than changes in stellar temperature, which has continued into the period covered by this paper. Individual measurements obtained from 2006 to 2011 are shown, together with the envelope of NIR variations from the literature (shaded) in Figure 5. The HiCIAO data were obtained at $H=6.74 \pm 0.08$, a historic minimum of NIR brightness for MWC 480. The constant temperature of the IR excess permits us to estimate the dust disk scale from the NIR excess. At the time of the HiCIAO observation, the disk scale height at the sublimation radius was only $\sim 60 \%$ of that seen in the 1998 data, which yielded a marginal detection of the disk. By contrast, Spitzer IRS and BASS observations made in 2004 corresponded to a maximum excess state. The HiCIAO data support an anticorrelation between disk detectability and the NIR excess, consistent with predictions for dust disks where grains have grown and settled to the disk midplane (Sitko et al. 2008; Wisniewski et al. 2008). 


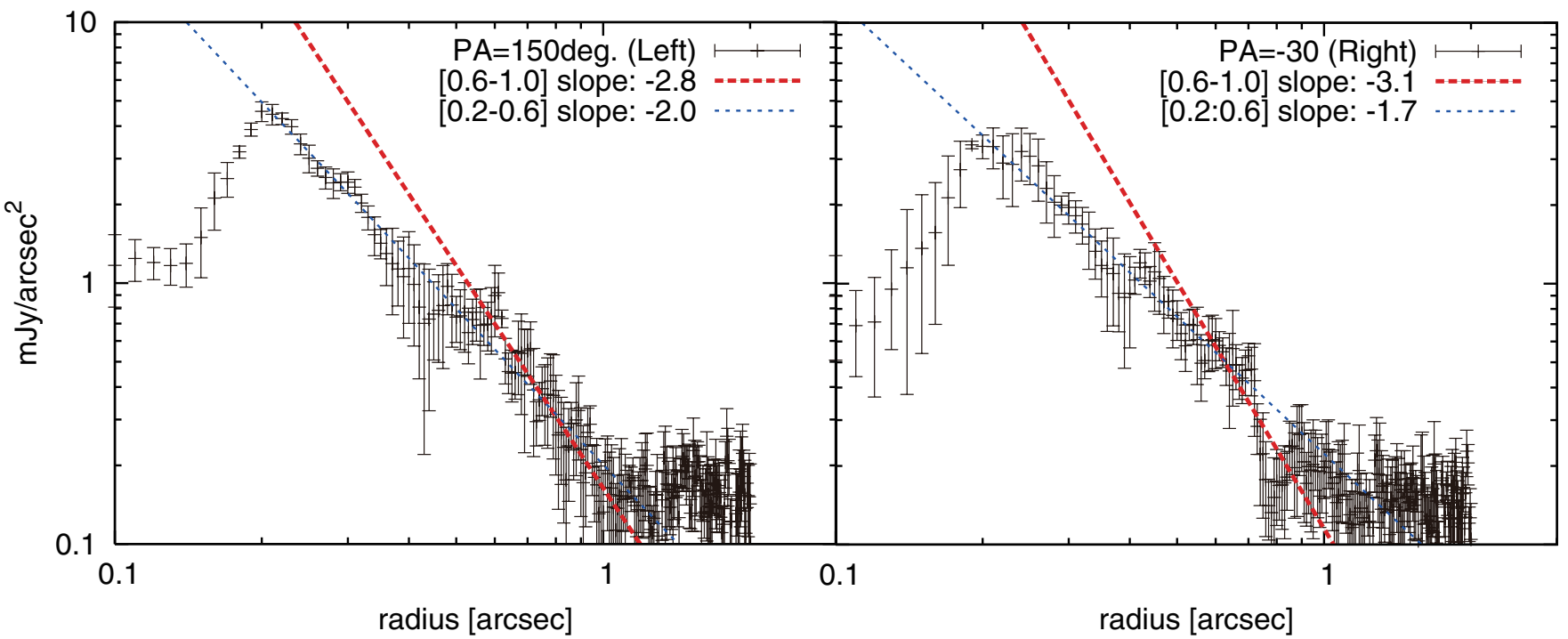

Figure 4. Polarization intensity radial profile along the major axis of MWC 480. Blue dashed lines show the profile from $0^{\prime \prime} 2$ to $0^{\prime \prime} 6$ of P.A. $=150^{\circ}\left(r^{-2.0 \pm 0.1}:\right.$ left $)$ and P.A. $=-30^{\circ}\left(r^{-1.7 \pm 0.1}\right.$ : right). Red dashed lines show the profile from $0^{\prime \prime} 6$ to $1^{\prime \prime} .0$ of P.A. $=150^{\circ}\left(r^{-2.8 \pm 0.2}\right.$ : left $)$ and P.A. $=-30^{\circ}\left(r^{-3.1 \pm 0.3}:\right.$ right $)$.

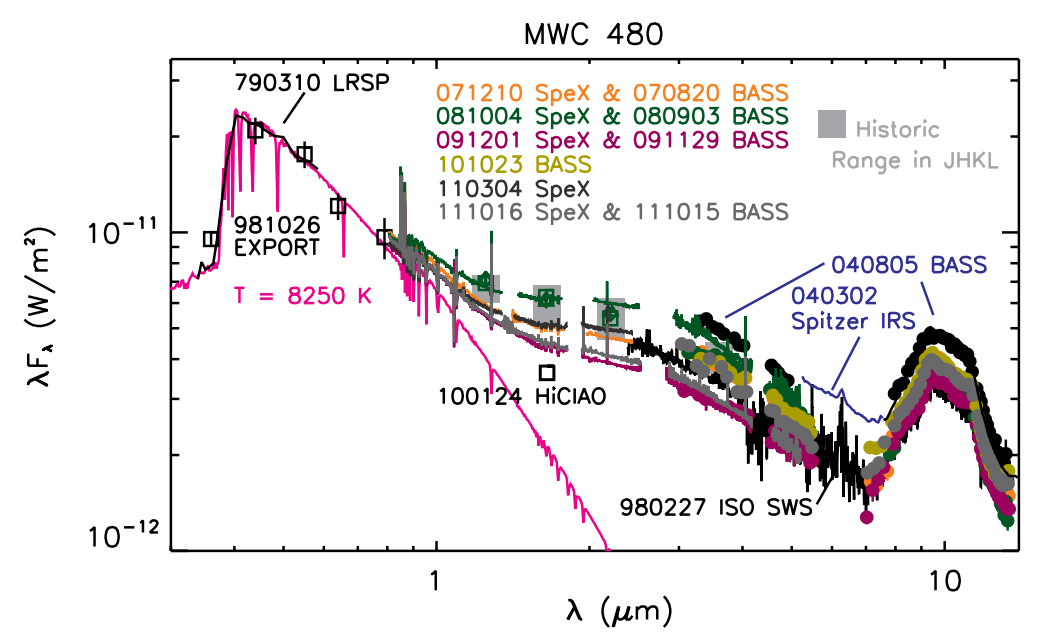

Figure 5. Spectral energy distribution of MWC 480. Multi-epoch data from BASS and SpeX along with the 2010 HiCIAO photometry are shown. Also shown are UBVRI photometry from the EXPORT consortium (Oudmaijer et al. 2001), data from the Low Resolution Spectrophotometer (LRSP) of the Pine Bluff Observatory (Sitko 1981), spectra from the Infrared Space Observatory (ISO) and the Spitzer Space Telescope, and a $T=8250$ K model atmosphere (Kurucz 1979). Note that the HiCIAO photometry data point is at a historic minimum as compared to the ensemble of other observations presented.

\section{DISCUSSION}

Despite being the brightest Herbig Ae star at millimeter wavelengths (Mannings et al. 1997), the disk of MWC 480 has proven elusive to imaging in scattered light. Despite multiple attempts with HST (Augereau et al. 2001; Grady et al. 2010) and Subaru/CIAO (Fukagawa et al. 2010), the disk has previously been imaged in scattered light only once, and then with only a marginal detection and incomplete recovery of the disk at all position angles (Grady et al. 2010). The HiCIAO PI image of this disk represents the first detection of the disk in polarized light, and a detection which moreover probes the disk at all position angles, except along the system semiminor axis. The successful detection of the disk relies on two factors. First, the HiCIAO observation was made at a time when the NIR excess was at a historic minimum, providing the most favorable illumination conditions for the outer disk. The second factor is polarimetric differential imaging with simultaneous measurement of the stellar PSF from the unpolarized stellar signal. The PI image was made by combining the Stokes $Q$ and $U$ images. The Stokes $Q$ and $U$ parameters were created by subtracting two split images on the HiCIAO image, which can subtract unpolarized light (PSF) of MWC 480 itself. This approach provides not only a perfect seeing match, but also an exact color match to the star.

Nevertheless, the disk is faint, with a fractional polarized intensity of only $\sim 0.1 \%$. Despite its relatively old age (7 Myr; Simon et al. 2000), the disk has yet to develop distinct structural features, including divots, rings, or a cavity. Our data do reveal what appears to be a break in the radial polarized intensity power law at $0^{\prime \prime} 6(84 \mathrm{AU})$, with a shallower power-law interior to that point. A similarly shallow power law is seen in the region of the spiral arms for SAO 206462 (Muto et al. 2012). However, the absence of distinct structure in the disk of MWC 480 constrains any spiral features in the disk to have a relative scale height of $(H / R)<0.05$, in order for them not to be detectable at the spatial resolution of the HiCIAO data. In tandem with the presence of ongoing accretion, which manifests itself through a soft X-ray spectrum, FUV excess light and mass loss via a jet (Grady et al. 2010), the disk of MWC 480 has a distinctly primordial character. 


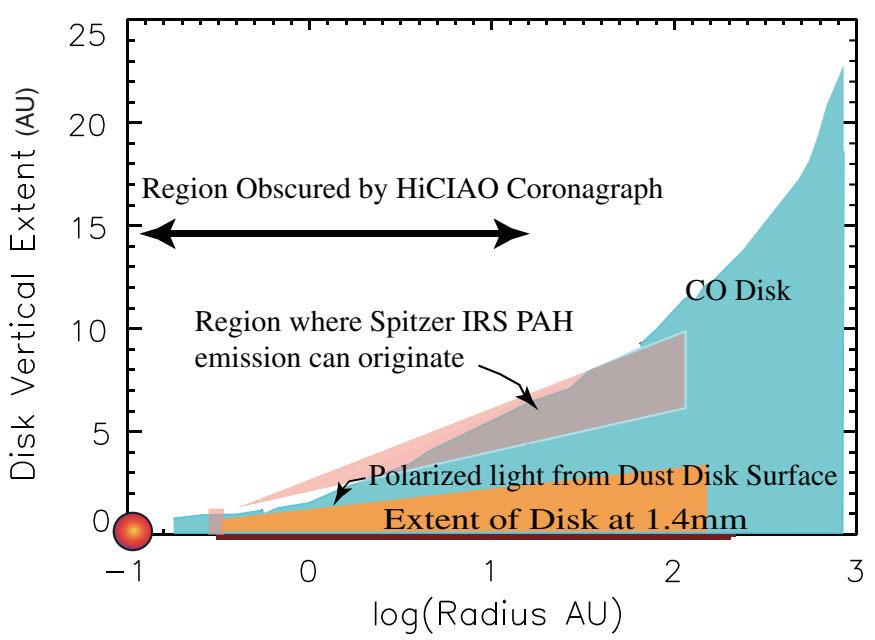

Figure 6. Schematic of the disk of MWC 480, based on data from the literature, and the geometrical constraints provided by the synoptic data and high-contrast imagery (brown: extent of disk at $1.4 \mathrm{~mm}$; orange: dust disk surface; light blue: CO disk; pink: possible Spitzer IRS PAH emission zones).

As befits a dynamically cold disk, MWC 480's dust disk is also geometrically quite thin. Our detection of the disk, in tandem with the previous NICMOS marginal detection, allows us to better constrain where the optically thick surface of the disk lies in the $H$ band relative to the gas disk. Piétu et al. (2007) found from modeling of $\mathrm{CO}$ interferometric data a $10 \pm 1.1 \mathrm{AU}$ gas disk scale height at $100 \mathrm{AU}$, corresponding to a disk opening half angle of $6^{\circ}$ and a disk inclination of 37.5 . Grady et al. (2010) estimated that the angle subtended by the dust disk rim at the sublimation radius was 2.2 for the 1998 NICMOS marginal detection of the disk in scattered light. For the HiCIAO data, the star was $0.5 \mathrm{mag}$ fainter at $\mathrm{H}$. If we assume that variation in the NIR excess light scales with changes in the scale height of the dust disk inner rim, then the rim subtended only $\sim 60 \%$ of the scale height seen in 1998 data. This constrains the optically thick dust disk surface to lie above 1.3. In tandem with the disk scattered light detection, this constrains the optically thick dust disk surface to lie above of 1.3. The available data therefore suggest that the surface of the dust disk lies between $1.3 \leqslant \theta \leqslant$ 2.2 . Adopting the average of this range, the dust disk has a scale height at 100 AU only $\sim 30 \%$ as high as the gas disk $(\mathrm{H} / \mathrm{R} \sim$ $0.03)$, demonstrating that this is a stratified disk. The synoptic data for MWC 480 further suggest that the outer dust disk is only rarely illuminated, in contrast to HD 163296 (Wisniewski et al. 2008; Fukagawa et al. 2010), although quantifying the duty cycle for disk illumination will require a denser grid of photometric and spectroscopic data than are currently available.

A further advantage of the availability of synoptic data in tandem with more specialized observations is that we can start to extract the vertical structure of the disk. By combining the imagery (NICMOS $1.1 \mu \mathrm{m}$ non-detection data; Grady et al. 2010) with the synoptic data, we find that the 2004 Spitzer IRS detection of polycyclic aromatic hydrocarbon $(\mathrm{PAH})$ emission in MWC 480 (Keller et al. 2008; Acke et al. 2010) corresponded to a high excess state (BASS data), a factor of three above the ISO SWS data (Figure 5). In this configuration, the entire dust disk surface would have been shadowed, indicating that PAH emission either originates from the inner rim of the dust disk or that it must be associated with material in the gas disk at altitude, rather than in close proximity to the outer dust disk (Figure 6). If extended to other data sets, this technique can allow us to understand which disk constituents are routinely exposed to UV radiation and which have less irradiation.

We can compare the outer disk properties of MWC 480 with the roughly coeval SAO 206462 and the younger, solar analog LkCa 15, both of which have similar PI measurements using HiCIAO (J. P. Wisniewski 2011, private communication; Thalmann et al. 2010; Muto et al. 2012) and prior HST imagery. Both MWC 480 and SAO 206462 have radial polarized intensity profiles beyond $0^{\prime \prime} 6$ proportional to $r^{-3}$ if the polarization efficiency is constant, suggesting that the outer disks are not highly flared. Model fits to the SED and submillimeter visibility for SAO 206462 indicate a disk scale height at the inner edge of the outer disk of 9.2 AU at $46 \mathrm{AU}$ (Andrews et al. 2011). To be illuminated at $100 \mathrm{AU}$, the dust disk in that system must lie above $20 \mathrm{AU}$, a factor of 6.7 times higher than that for MWC 480. As for LkCa 15, the NICMOS data demonstrate that the disk is detected out to 1".2 (G. Schneider 2011, private communication), with no evidence of shadowing of the outer disk along the system major axis. To meet this illumination condition, assuming a wall height of $7 \mathrm{AU}$ at $50 \mathrm{AU}$ in radius (Andrews et al. 2011), the dust disk of LkCa 15 must extend more than $14 \mathrm{AU}$ above the midplane at $100 \mathrm{AU}$ in radius. The combination of a planet candidate in the disk of LkCa 15 (Kraus \& Ireland 2012), and spiral arms which may also be associated with Jovian-mass planets in SAO 206462 (Muto et al. 2012), suggests that the outer disks of these transitional disk systems are dynamically excited by massive planets. The extent to which this process is present may therefore constrain the masses of planets, even for systems where the inner cavity is inaccessible to current imaging technology. However, the primordial character of MWC 480's disk compared to coeval or even younger systems demonstrates that the process of giant planet formation may not be ubiquitous or proceed at the same rate in all systems. Addressing how the planet hosting disks differ from those with no evidence of planet formation will be a major task both for ALMA and for the next generation of high-contrast imagers.

\section{SUMMARY}

We report the detection of the disk of the Herbig Ae star, MWC 480, in scattered, polarized light from 0.2 to $1^{\prime \prime} .0$ (28-137 AU) in data taken with HiCIAO at the Subaru $8.2 \mathrm{~m}$ telescope in early 2010. At the epoch of the detection, the NIR excess was at a historic low. Given a history of excess amplitude but not temperature changes, this implies that the vertical extent of the inner rim of the dust disk, and by inference the accretion rate onto the star, was at a historic low. An anticorrelation between the scale height of the dust disk near the sublimation radius and the degree of illumination of the outer disk is expected only when the dust disk has settled toward the disk midplane. The data presented here, in tandem with earlier synoptic photometry and spectroscopy and a history of high-contrast imaging observations, provide direct confirmation that Herbig Ae stars with IR excesses characterized by power laws of their $\mathrm{H} / \mathrm{R}$ are disks where dust grains have grown, settled, and where the outer disk is largely shadowed. Higher cadence synoptic observations are required to quantify the illumination/shadowing duty cycle for MWC 480 and to obtain sufficient data for other Meeus group II disks to establish whether MWC 480 is typical of the shadowed disks. The first steps in comparing the disk geometry of systems like MWC 480 to older, transitional disks have demonstrated that the transitional disks considered have not only wide gaps, but outer disks which are much more vertically extended than seen 
for MWC 480. As the SEEDS survey continues, we will have the opportunity to obtain data not only for other transitional disks, but also for additional primordial disks, and should be able to test the hypothesis that transitional disks have structural changes that extend over the entire disk, reflecting the presence of giant planets within the gapped region.

This work is partially supported by KAKENHI 22000005 (M.T.), KAKENHI 23103004, 23740151 (M.F.), NSF AST 1008440 (C.A.G.), NSF AST 1009314 (J.P.W.), NASA NNX09AC73G (C.A.G. and M.L.S.), and the IR\&D program at The Aerospace Corporation (R.W.R.).

\section{REFERENCES}

Acke, B., Bouwman, J., Juhász, A., et al. 2010, ApJ, 718, 558

Acke, B., Min, M., van den Ancker, M. E., et al. 2009, A\&A, 502, L17

Andrews, S. M., Wilner, D. J., Espaillat, C., et al. 2011, ApJ, 732, 42

Augereau, J.-C., Lagrange, A. M., Mouillet, D., \& Ménard, F. 2001, A\&A, 365, 78

Carciofi, A. C., Bjorkman, J. E., \& Magalhães, A. M. 2004, ApJ, 604, 238

Cushing, M. C., Vacca, W. D., \& Rayner, J. T. 2004, PASP, 116, 362

Cutri, R. M., et al. 2003, Explanatory Supplement to the 2MASS All Sky Data

Release (Washington, DC: NASA), http:/www.ipac.caltech.edu/2mass/ releases/allsky/doc/explsup.html

Dullemond, C. P., \& Dominik, C. 2004a, A\&A, 417, 159

Dullemond, C. P., \& Dominik, C. 2004b, A\&A, 421, 1075

Fukagawa, M., Tamura, M., Itoh, Y., et al. 2010, PASJ, 62, 347

Grady, C. A., Devine, D., Woodgate, B., et al. 2000, ApJ, 544, 895

Grady, C. A., Hamaguchi, K., Schneider, G., et al. 2010, ApJ, 719, 1565

Grady, C. A., Polomski, E. F., Henning, T., et al. 2001, AJ, 122, 3396

Grady, C. A., Schneider, G., Hamaguchi, K., et al. 2007, ApJ, 665, 1391

Grady, C. A., Woodgate, B. E., Bowers, C. W., et al. 2005, ApJ, 630, 958

Hackwell, J. H., Warren, D. W., Chatelain, M. A., Dotan, Y., \& Li, P. H 1990, Proc. SPIE, 1235, 171

Hashimoto, J., Tamura, M., Muto, T., et al. 2011, ApJ, 729, L17

Hawarden, T. G., Leggett, S. K., Letawsky, M. B., Ballantyne, D. R., \& Casali, M. M. 2001, MNRAS, 325, 563
Hayano, Y., Takami, H., Guyon, O., et al. 2008, Proc. SPIE, 7015E, 701510

Hinkley, S., Oppenheimer, B. R., Soummer, R., et al. 2009, ApJ, 701, 804

Joos, F., Buenzli, E., Schmid, H. M., et al. 2008, Proc. SPIE, 7016, 48

Keller, L. D., Sloan, G. C., Forrest, W. J., et al. 2008, ApJ, 684, 411

Kóspál, Á., Prusti, T., Cox, N. L. J., et al. 2012, A\&A, 541, 71

Kraus, A. L., \& Ireland, M. J. 2012, ApJ, 745, 19

Kurucz, R. L. 1979, ApJS, 40, 1

Leggett, S. K., Currie, M. J., Varricatt, W. P., et al. 2006, MNRAS, 373, 781

Mannings, V., Koerner, D., \& Sargent, A. 1997, Nature, 388, 555

Meeus, G., Waters, L. B. F. M., Bouwman, J., et al. 2001, A\&A, 365, 476

Min, M., Canovas, H., Mulders, G. D., \& Keller, C. U. 2012, A\&A, 537, A75

Muto, T., Grady, C. A., Hashimoto, J., et al. 2012, ApJ, 748, L22

Oudmaijer, R. D., Palacios, J., Eiroa, C., et al. 2001, A\&A, 379, 564

Piétu, V., Dutrey, A., \& Guilloteau, S. 2007, A\&A, 467, 163

Piétu, V., Dutrey, A., Guilloteau, S., Chapillon, E., \& Pety, J. 2006, A\&A, 460, L43

Quanz, S. P., Schmid, H. M., Geissler, K., et al. 2011, ApJ, 738, 23

Rayner, J. T., Cushing, M. C., \& Vacca, W. D. 2009, ApJS, 185, 289

Rayner, J. T., Toomey, D. W., Onaka, P. M., et al. 2003, PASP, 115, 362

Rebull, L. M. 2011, in ASP Conf. Ser. 448, 16th Cambridge Workshop on Cool Stars, Stellar Systems, and the Sun, ed. C. M. Johns-Krull, M. K. Browning, \& A. A. West (San Francisco, CA: ASP), 5

Robitaille, T., Whitney, B. A., Indebetouw, R., \& Wood, K. 2007, ApJS, 169, 328

Simon, M., Dutrey, A., \& Guilloteau, S. 2000, ApJ, 545, 1034

Simons, D. A., \& Tokunaga, A. 2002, PASP, 114, 169

Sitko, M. L. 1981, ApJ, 247, 1024

Sitko, M. L., Carpenter, W. J., Kimes, R. L., et al. 2008, ApJ, 678, 1070

Skrutskie, M. F., Cutri, R. M., Stiening, R., et al. 2006, AJ, 131, 1163

Tamura, M. 2009, in AIP Conf. Ser. 1158, Exoplanets and Disks: Their Formation and Diversity, ed. T. Usuda, M. Tamura, \& M. Ishii (Melville, NY: AIP), 11

Tamura, M., Hodapp, K., Takami, H., et al. 2006, Proc. SPIE, 6269, 62690V

Tanii, R., et al. 2012, PASJ, submitted

Thalmann, C., Grady, C. A., Goto, M., et al. 2010, ApJ, 718, L87

Tokunaga, A. T., Simons, D. A., \& Vacca, W. D. 2002, PASP, 114, 180

Tokunaga, A. T., \& Vacca, W. D. 2005, PASP, 117, 421

Vacca, W. D., Cushing, M. C., \& Rayner, J. T. 2003, PASP, 115, 389

van Leeuwen, F. 2007, A\&A, 474, 653

Wisniewski, J. P., Clampin, M., Grady, C. A., et al. 2008, ApJ, 682, 548 